\title{
On two problems regarding the Hamiltonian cycle game
}

\author{
Dan Hefetz \\ Institute of Theoretical Computer Science \\ ETH Zurich, CH-8092 Switzerland \\ dan.hefetz@inf .ethz.ch
}

\author{
Sebastian Stich \\ Department of Mathematics \\ ETH Zurich, CH-8092 Switzerland \\ sstich@student.ethz.ch
}

Submitted: Oct 24, 2008; Accepted: Feb 18, 2009; Published: Feb 27, 2009

Mathematics Subject Classification: 91A46, 05C45

\begin{abstract}
We consider the fair Hamiltonian cycle Maker-Breaker game, played on the edge set of the complete graph $K_{n}$ on $n$ vertices. It is known that Maker wins this game if $n$ is sufficiently large. We are interested in the minimum number of moves needed for Maker in order to win the Hamiltonian cycle game, and in the smallest $n$ for which Maker has a winning strategy for this game.

We prove the following results: (1) If $n$ is sufficiently large, then Maker can win the Hamiltonian cycle game within $n+1$ moves. This bound is best possible and it settles a question of Hefetz, Krivelevich, Stojaković and Szabó; (2) If $n \geq 29$, then Maker can win the Hamiltonian cycle game. This improves the previously best bound of 600 due to Papaioannou.
\end{abstract}

\section{Introduction}

Let $\mathcal{F}$ be a hypergraph. In the fair Maker-Breaker game $\mathcal{F}$ two players, called Maker and Breaker, take turns in claiming previously unclaimed vertices of $\mathcal{F}$, with Breaker going first. Each player claims one vertex per turn. Maker wins the game as soon as he claims all the vertices of some hyperedge of $\mathcal{F}$. If by the time every vertex of $\mathcal{F}$ is claimed by some player, Maker was not able to fully claim any hyperedge of $\mathcal{F}$, then Breaker wins the game. The game which differs from $\mathcal{F}$ only in the fact that Maker is the first player instead of Breaker, will be denoted by $\mathcal{F}^{M}$.

Let $n$ be a positive integer, and let $\mathcal{H}_{n}$ be the hypergraph whose vertices are the edges of $K_{n}$, and whose hyperedges are the edge sets of all Hamiltonian cycles of $K_{n}$. In this paper we study the fair Maker-Breaker Hamiltonian cycle game $\mathcal{H}_{n}$. 
Following [2], we define $\tau\left(\mathcal{H}_{n}\right)$ to be the minimum number of moves needed for Maker in order to win $\mathcal{H}_{n}$ (it follows from a result of Chvátal and Erdős [1] that $\tau\left(\mathcal{H}_{n}\right)<\infty$ for sufficiently large $n)$. It was proved in [2] that $n+1 \leq \tau\left(\mathcal{H}_{n}\right) \leq n+2$ holds for sufficiently large $n$. It was also asked there, which of these two values is the correct answer. We settle this question as follows:

Theorem 1.1 For sufficiently large $n$ we have $\tau\left(\mathcal{H}_{n}\right)=n+1$.

Note that, though this only improves the upper bound given in [2] by 1, the proof is much more involved.

Another problem we address in this paper, is that of determining the smallest $n$ for which Maker has a winning strategy in $\mathcal{H}_{n}^{M}$. Though it seems plausible, it is not known that if Maker wins $\mathcal{H}_{n}^{M}$ for some integer $n$, then he also wins $\mathcal{H}_{n+1}^{M}$. Hence, we define $\kappa\left(\mathcal{H}_{n}^{M}\right)$ to be the smallest positive integer $n_{0}$ such that Maker can win $\mathcal{H}_{n}^{M}$ for every $n \geq n_{0}$. Papaioannou [4] proved that $\kappa\left(\mathcal{H}_{n}^{M}\right) \leq 600$, and conjectured that in fact $\kappa\left(\mathcal{H}_{n}^{M}\right)=8$. We improve his upper bound as follows:

Theorem $1.2 \kappa\left(\mathcal{H}_{n}^{M}\right) \leq 29$.

\section{Preliminaries}

Our graph-theoretic notation is standard and follows that of [6]. In particular, we use the following: for a graph $G$, denote its set of vertices by $V(G)$, and its set of edges by $E(G)$. Moreover, let $v(G)=|V(G)|$ and $e(G)=|E(G)|$. For a graph $G=(V, E)$ and a set $A \subseteq V$ denote by $G[A]$ the subgraph of $G$ induced by $A$.

We will also need the following more specific terminology. An edge that was not previously claimed by either player is called free. A graph is called a linear forest if each of its connected components is a non-empty path. Let $S \subseteq V\left(K_{n}\right)$ be an arbitrary set. Assume that just before Maker's $(k+1)$ st move, the subgraph of his graph which is induced on the vertices of $V\left(K_{n}\right) \backslash S$, admits a linear forest. Let $B_{k}^{\prime}(S)$ denote the current subgraph

of Breaker's graph on $K_{n} \backslash S$, which is induced on the endpoints of Maker's paths and on the vertices which are isolated in Maker's graph. Let $B_{k}(S)$ be the subgraph of $B_{k}^{\prime}(S)$ which is obtained by removing all edges $(x, y)$ such that $x$ and $y$ are endpoints of the same path of Maker. Let $B_{k}^{+}(S)$ denote the graph whose vertex set is $V\left(B_{k}(S)\right) \cup S$ and whose edges are the edges of $B_{k}(S)$ and the edges of Breaker that connect a vertex of $S$ and a vertex of $B_{k}(S)$. We abbreviate $B_{k}(\emptyset)$ to $B_{k}$.

Let $G_{k}^{\prime}(S)$ denote the subgraph of $K_{n}$ which is induced on the vertices of $B_{k}(S)$. Let $G_{k}(S)$ be the subgraph of $G_{k}^{\prime}(S)$ which is obtained by removing all edges $(x, y)$ such that $x$ and $y$ are endpoints of the same path of Maker. The free edges of $G_{k}(S)$ are called available. 
The rest of the paper is organized as follows. In Section 3 we prove Theorem 1.1 and in Section 4 we prove Theorem 1.2. Finally, in Section 5 we present some concluding remarks and related open problems.

\section{Winning the Hamiltonian cycle game quickly}

In this section we prove Theorem 1.1. In order to do so, we will first present a strategy for Maker, then show that, using this strategy, Maker can win $\mathcal{H}_{n}$ in $n+1$ moves, and finally prove that Maker can indeed follow this strategy. Throughout this section we assume that $n$ is as large as necessary.

\subsection{Maker's strategy}

Phase 1. Maker starts by building two vertex disjoint paths, $P=p_{0} p_{1} \ldots p_{5}$ and $Q=$ $q_{0} q_{1} \ldots q_{5}$, such that just after his 10-th move, the following properties hold:

(i) every edge of Breaker is incident with some vertex of $P \cup Q$;

(ii) at least one of the two edges $\left(p_{0}, p_{5}\right)$ and $\left(q_{0}, q_{5}\right)$ is free.

If possible, Maker claims either $\left(p_{0}, p_{5}\right)$ or $\left(q_{0}, q_{5}\right)$ in his 11 th move and continues to Phase X; otherwise he proceeds to Phase A.

Phase A. If there are no edges of Breaker that connect an endpoint of one of Maker's paths and some vertex of one of his other paths, then Maker goes directly to Phase B.

Otherwise he chooses an arbitrary endpoint $v$ of one of his paths which is incident with such an edge of Breaker, and claims a free edge that is incident with $v$ and some arbitrary isolated vertex. If he can now close this longer path into a cycle in his next move, then he does so and continues to Phase X. Otherwise he repeats Phase A.

Phase B. If every vertex which is of positive degree in Breaker's graph belongs to some path of Maker, then Maker goes directly to Phase 2.

Otherwise, let $v$ be an arbitrary vertex that is isolated in Maker's graph but not in Breaker's graph. In his next move, Maker claims a free edge that connects $v$ and an endpoint of one of his paths, such that the edge that connects $v$ and the other endpoint of that path is still free. If he can now close this longer path into a cycle in his next move, then he does so and continues to Phase X. Otherwise he repeats Phase B. 
Phase 2. If Maker's graph consists of at least 15 vertex disjoint paths, then he proceeds to Phase 3.

Otherwise he builds a new path $P=p_{0} p_{1} \ldots p_{5}$ which is vertex disjoint from all of his other paths, such that just after building $P$ the following properties hold:

(i) every edge of Breaker is incident with a vertex of some path of Maker;

(ii) the edge $\left(p_{0}, p_{5}\right)$ is free.

If Maker can close the new path $P$ into a cycle in his next move, then he does so and continues to Phase X. Otherwise he proceeds to Phase A.

Phase 3. If there are at most 9 isolated vertices in Maker's graph, then he goes directly to Phase 4.

Otherwise he makes one of his paths (chosen arbitrarily) longer, by claiming in his next move a free edge that connects an endpoint of this path and an arbitrary isolated vertex. If he can now close this longer path into a cycle in his next move, then he does so and continues to Phase X. Otherwise he repeats Phase 3.

Phase 4. Let $I$ denote the set of isolated vertices in Maker's graph. First, Maker builds a Hamiltonian path $P$ on $K_{n}[I]$ (this is not a trivial task, the exact details of how Maker can achieve this goal are given in Lemma 3.3). Then, he connects an endpoint of $P$ to an endpoint of some other path of his, such that the edge that connects the endpoints of the new path is still free. If he can now close this longer path into a cycle in his next move, then he does so and continues to Phase X. Otherwise he proceeds to Phase 5.

Phase 5. If Maker's graph consists of exactly 2 paths, then he goes directly to Phase 6 . Otherwise, in his next move, Maker claims an edge $e=(x, y)$ such that the following properties hold:

(i) $x$ and $y$ are endpoints of two distinct paths of Maker, denoted by $P_{x}$ and $P_{y}$ respectively;

(ii) the edge $\left(x^{\prime}, y^{\prime}\right)$, where $x^{\prime} \neq x$ is an endpoint of $P_{x}$ and $y^{\prime} \neq y$ is an endpoint of $P_{y}$, is free;

(iii) if there exists an edge of Breaker that connects endpoints of two different paths of Maker, then $(x, y)$ is adjacent to at least one such edge.

If Maker can now close this longer path into a cycle in his next move, then he does so and continues to Phase X. Otherwise he repeats Phase 5.

Phase 6. Maker uses 3 more moves to turn his two paths into a Hamiltonian cycle (the exact details of how Maker can achieve this goal are given in Lemma 3.6). 
Phase X. Let $C$ denote Maker's cycle. Maker builds a Hamiltonian path $P$ on $K_{n} \backslash C$, and then connects $P$ and $C$ to form a Hamiltonian cycle in $K_{n}$ (the exact details of how Maker can achieve these goals are given in Lemma 3.3 and Lemma 3.7). This phase lasts exactly $n+1-k_{X}$ moves, where $k_{X}$ denotes the number of moves that Maker has made before entering Phase X.

\subsection{Proof of correctness}

It is clear that if Maker can play according to this strategy, then he wins $\mathcal{H}_{n}$ in $n+1$ moves. Hence, it suffices to prove that Maker can indeed follow his strategy. Before doing so, we state and prove some auxiliary lemmas.

The first phase of Maker's strategy consists of building two vertex disjoint paths. The following lemma asserts that he can indeed do so.

Lemma 3.1 Let $s \geq 2$ and let $n>4(s+1)$. Playing on $K_{n}$, Maker, as the second player, has a strategy for building two vertex disjoint paths $P=p_{0} p_{1} \ldots p_{s}$ and $Q=q_{0} q_{1} \ldots q_{s}$, such that just after his (2s)-th move the following properties hold:

(i) every edge of Breaker is incident with some vertex of $P \cup Q$;

(ii) at least one of the two edges $\left(p_{0}, p_{s}\right)$ and $\left(q_{0}, q_{s}\right)$ is free.

Proof We will prove this lemma by induction on the number of moves made by Maker.

For $1 \leq i \leq 2$ and $2 \leq j \leq 2 s$, let $P_{i}^{j}$ denote the $i$-th path of Maker, just after his $j$-th move. Let $p_{i}^{j \prime}$ and $p_{i}^{j \prime \prime}$ denote the endpoints of $P_{i}^{j}$. Maker's goal while building his paths is to ensure that, for every $1 \leq i \leq 2$ and $2 \leq j \leq 2 s$, the following properties hold after his $j$-th move.

(a) If $v\left(P_{i}^{j}\right)<s+1$, then $p_{i}^{j \prime}$ is incident with at most one edge of Breaker which is not already incident with another vertex of $P_{1}^{j} \cup P_{2}^{j}$.

(b) If $v\left(P_{i}^{j}\right)<s+1$, then $p_{i}^{j \prime \prime}$ is not incident with any edge of Breaker which is not incident with another vertex of $P_{1}^{j} \cup P_{2}^{j}$.

(c) Every edge of Breaker is incident with a vertex of $P_{1}^{j} \cup P_{2}^{j}$.

(d) At least one of the edges $\left(p_{1}^{j \prime}, p_{1}^{j \prime \prime}\right)$ and $\left(p_{2}^{j \prime}, p_{2}^{j \prime \prime}\right)$ does not belong to Breaker.

In his first two moves, Maker claims two independent edges $e$ and $f$ such that, when viewed as paths $P_{1}^{2}=e, P_{2}^{2}=f$, they satisfy (a), (b), (c) and (d); it is straightforward to verify that this can be done. 
Assume that properties (a), (b), (c) and (d) hold after Maker's $j$-th move, for some $2 \leq j<2 s$. Let $g=\left(g^{\prime}, g^{\prime \prime}\right)$ denote the edge claimed by Breaker in his $(j+1)$ st move. We distinguish between the following three cases.

Case 1: $g$ is not incident with any vertex of $P_{1}^{j} \cup P_{2}^{j}$. Assume without loss of generality that the length of $P_{1}^{j}$ is strictly less than $s$ (otherwise this holds for $P_{2}^{j}$ ). By property (a), at least one of the edges $\left(g^{\prime}, p_{1}^{j \prime}\right),\left(g^{\prime \prime}, p_{1}^{j \prime}\right)$ is still free. In his next move, Maker claims one of these edges, entailing (a), (b), (c) and (d) for $P_{1}^{j+1}$ and $P_{2}^{j+1}$.

Case 2.a: $g$ is incident with $P_{1}^{j}$, but not with $P_{2}^{j}$. If $P_{1}^{j}$ is already of length $s$, then Maker claims an edge that connects $p_{2}^{j \prime \prime}$ and an arbitrary isolated vertex. Such an isolated vertex exists by property (c), and since $n>4(s+1)$. Henceforth we assume that $v\left(P_{1}^{j}\right)<s+1$.

If $g$ is incident with both endpoints of $P_{1}^{j}$, then Maker claims an edge that connects $p_{1}^{j \prime \prime}$ and an arbitrary isolated vertex.

If $g$ is incident with exactly one endpoint of $P_{1}^{j}$, then Maker claims an edge between this endpoint and an arbitrary isolated vertex.

If $g$ is not incident with any endpoint of $P_{1}^{j}$, then Maker claims an edge that connects $p_{1}^{j \prime \prime}$ and an arbitrary isolated vertex.

Clearly, after this move, (a), (b), (c) and (d) will hold for $P_{1}^{j+1}$ and $P_{2}^{j+1}$.

Case 2.b: $g$ is incident with $P_{2}^{j}$, but not with $P_{1}^{j}$. This case can be treated similarly to Case 2.a.; we omit the straightforward details.

Case 3: $g$ is incident with both paths $P_{1}^{j}$ and $P_{2}^{j}$. Maker plays as in Case 2 for $P_{i}^{j}$, where $1 \leq i \leq 2$ is the smallest integer such that the length of $P_{i}^{j}$ is strictly smaller than $s$.

Properties $(i)$ and (ii) are clearly satisfied after Maker's (2s)-th move, as he maintained properties $(c)$ and $(d)$ respectively.

This concludes the proof of Lemma 3.1.

The following lemma asserts that Maker can continue to build more paths.

Lemma 3.2 Assume that some edges of $K_{n}$ were already claimed by the players. Let $r$ be a positive integer, and let $S$ denote the set of vertices of positive degree in Maker's graph. If all of the following properties hold:

(a) Maker is next to play;

(b) every edge of Breaker is incident with some vertex of $S$;

(c) $n-|S|>2(r+1)$; 
then Maker can build a path $P=p_{0} p_{1} \ldots p_{r}$, such that just after his $r$-th move the following properties hold:

(i) $P \cap S=\emptyset$;

(ii) every edge of Breaker is incident with some vertex of $P \cup S$;

(iii) the edge connecting the endpoints of $P$ is free.

The proof of Lemma 3.2 is very similar to that of Lemma 3.1. We omit the straightforward details.

A central ingredient of Maker's strategy is building a long path with some additional properties. This is taken care of by the following lemma.

Lemma 3.3 Let $S \subseteq V\left(K_{n}\right)$ be an arbitrary subset. Assume that just before Maker's $\left(k_{0}+1\right)$ st move, the edges with both endpoints in $K_{n} \backslash S$ he has claimed so far, span a linear forest $F$, consisting of $f$ paths. Let $I=V\left(K_{n} \backslash F\right) \backslash S$, and let $e_{b}$ denote the number of Breaker's edges that are not incident with any vertex of $S \cup V(F)$. If either

(i) $|I| \geq 9, e_{b} \leq 2$ and $e\left(B_{k_{0}}^{+}(S)\right) \leq 2 f+\left\lceil\frac{|I|}{2}\right\rceil-6$; or

(ii) $I=\emptyset$ and $e\left(B_{k_{0}}^{+}(S)\right) \leq 2 f-3$;

then Maker can build a Hamiltonian path $P$ on $K_{n} \backslash S$ in $f+|I|-1$ moves, while ensuring that $e\left(B_{k_{0}+f+|I|-1}^{+}(S)\right) \leq 3$.

The proof of Lemma 3.3 will rely on the following two lemmas:

Lemma 3.4 Let $e_{1}$ and $e_{2}$ be two arbitrary edges of $K_{m}$. Let $\mathcal{F}_{m}\left(e_{1}, e_{2}\right)$ denote the hypergraph whose vertices are the edges of $K_{m} \backslash\left\{e_{1}, e_{2}\right\}$ and whose hyperedges are the spanning linear forests of $K_{m} \backslash\left\{e_{1}, e_{2}\right\}$. If $m \geq 9$, then

$$
\tau\left(\mathcal{F}_{m}^{M}\left(e_{1}, e_{2}\right)\right)=\left\lfloor\frac{m}{2}\right\rfloor+1 .
$$

Proof For the sake of convenience, we will assume that both $e_{1}$ and $e_{2}$ were claimed by Breaker.

Assume first that $m$ is even. In the following, Maker ensures that for every $1 \leq k<\frac{m}{2}$, the following two properties hold after his $k$-th move:

(i) Maker's graph is a matching consisting of $k$ edges.

(ii) Every edge of Breaker is adjacent to some edge of Maker. 
First note, that if these properties hold for $k=\frac{m}{2}-1$, then Maker can build the spanning linear forest within two additional moves. This follows easily since there are only two isolated vertices in Maker's graph, Breaker has claimed $2+\frac{m}{2}-1$ edges (including $e_{1}$ and $\left.e_{2}\right)$, and $m \geq 9$.

It remains to show that Maker can indeed maintain properties (i) and (ii) for every $1 \leq k<\frac{m}{2}$. For $k=1$ this is clear, as Maker can claim an edge that is adjacent to both $e_{1}$ and $e_{2}$. Assume now that (i) and (ii) hold for some $1 \leq k<\frac{m}{2}-1$. In his next move, Breaker claims some edge $e$. If $e$ is adjacent to some edge of Maker, then he claims an arbitrary edge that connects two vertices that are isolated in his graph. If $e$ is not adjacent to any edge of Maker, then he claims an edge which is adjacent to $e$ and which connects two vertices that are isolated in his graph. It follows that properties (i) and (ii) hold after his $(k+1)$ st move. In both cases, the required edge exists, because there are $m-2 k>3$ isolated vertices in Maker's graph, and, by property (ii), every edge of Breaker (except possibly $e$ ) is adjacent to some edge of Maker.

If $m$ is odd, then Maker's strategy is essentially the same; we omit the straightforward details.

Lemma 3.5 Assume that just before Maker's $\left(k_{0}+1\right)$ st move, his graph admits a linear forest $F$. Let $f \geq 1$ denote the number of paths in $F$, and let $S=V\left(K_{n} \backslash F\right)$. If $e\left(B_{k_{0}}^{+}(S)\right) \leq v\left(B_{k_{0}}(S)\right)-3$, then Maker can build a Hamiltonian path on $K_{n} \backslash S$ in $f-1$ moves, while ensuring that $e\left(B_{k_{0}+f-1}^{+}(S)\right) \leq 3$.

Proof In each of his moves, Maker connects two endpoints of two different paths of his graph on $K_{n} \backslash S$. Hence, after each move of Maker, the number of paths in his graph decreases by one, and thus, after $f-1$ moves, he will build a Hamiltonian path on $K_{n} \backslash S$. Of course one has to prove that this is indeed possible; that is, to prove that there will always be an available edge for Maker to claim. In order to ensure this, for every $k_{0} \leq k \leq k_{0}+f-2$, Maker will claim an available edge while making sure that at least one of the following properties is satisfied:

(a) $e\left(B_{k}^{+}(S)\right) \leq v\left(B_{k}(S)\right)-3$;

(b) $e\left(B_{k}(S)\right) \leq 3$ and $e\left(B_{k}^{+}(S)\right) \leq v\left(B_{k}(S)\right)+1$.

We prove by induction on $k$ that this is indeed possible, and guarantees the existence of an available edge just before Maker's $(k+1)$ st move.

For $k=k_{0}$, property (a) holds because of the assumption of the lemma.

Let $k_{0} \leq k<k_{0}+f-2$; this ensures that $v\left(B_{k}(S)\right) \geq 6$. Assume first that property $(b)$ holds for $k$; we prove that it will hold for $k+1$ as well. Since property $(b)$ holds for $k$ and $v\left(B_{k}(S)\right) \geq 6$, it follows that for every $v \in V\left(G_{k}(S)\right)$, there exists an available edge which 
is incident with $v$. Maker plays as follows (note that the motivation behind certain parts of his strategy described here, will become clear only when we consider his last move):

Case 1: $e\left(B_{k}(S)\right)=3$. If $e\left(B_{k}^{+}(S) \backslash B_{k}(S)\right)=0$, then Maker claims an arbitrary available edge which is incident with some edge of $B_{k}(S)$. It follows that $e\left(B_{k+1}(S)\right) \leq 3$ and $e\left(B_{k+1}^{+}(S)\right) \leq e\left(B_{k}^{+}(S)\right)-1+1 \leq 3 \leq v\left(B_{k}(S)\right)-1=v\left(B_{k+1}(S)\right)+1$ after Breaker's next move. Hence, property $(b)$ will hold for $k+1$. Hence, we can assume that $e\left(B_{k}^{+}(S) \backslash B_{k}(S)\right)>0$.

If there exists a vertex of $V\left(B_{k}(S)\right)$ which has degree at least 2 in $B_{k}^{+}(S)$, then Maker claims an available edge $(x, y)$ which is incident with such a vertex, is adjacent to some edge of $B_{k}(S)$, is adjacent to at least 3 edges of $B_{k}^{+}(S)$, and maximizes $\operatorname{deg}_{B_{k}^{+}(S)}(x)+\operatorname{deg}_{B_{k}^{+}(S)}(y)$ amongst all such edges. It is easy to see that this is possible since $e\left(B_{k}(S)\right)=3$ and $e\left(B_{k}^{+}(S)\right) \geq 4$. It follows that $e\left(B_{k+1}(S)\right) \leq 3$ and $e\left(B_{k+1}^{+}(S)\right) \leq e\left(B_{k}^{+}(S)\right)-3+1 \leq v\left(B_{k}(S)\right)-1=v\left(B_{k+1}(S)\right)+1$ after Breaker's next move. Hence, property $(b)$ will hold for $k+1$.

Otherwise, every vertex of $B_{k}(S)$ has degree at most 1 in $B_{k}^{+}(S)$, and therefore $e\left(B_{k}^{+}(S)\right) \leq v\left(B_{k}(S)\right)-3$. Hence, Maker claims an arbitrary available edge which is adjacent to some edge of $B_{k}(S)$. It follows that $e\left(B_{k+1}(S)\right) \leq 3$ and $e\left(B_{k+1}^{+}(S)\right) \leq$ $e\left(B_{k}^{+}(S)\right)-1+1 \leq v\left(B_{k}(S)\right)-1=v\left(B_{k+1}(S)\right)+1$ after Breaker's next move. Hence, property $(b)$ will hold for $k+1$.

Case 2: $0 \leq e\left(B_{k}(S)\right) \leq 2$. If there exists an available edge which is adjacent to at least three edges of $B_{k}^{+}(S)$, then Maker claims an edge $(x, y)$ that maximizes $\operatorname{deg}_{B_{k}^{+}(S)}(x)+$ $\operatorname{deg}_{B_{k}^{+}(S)}(y)$ amongst all such edges. It follows that $e\left(B_{k+1}(S)\right) \leq 3$ and $e\left(B_{k+1}^{+}(S)\right) \leq$ $e\left(B_{k}^{+}(S)\right)-3+1 \leq v\left(B_{k}(S)\right)-1=v\left(B_{k+1}(S)\right)+1$ after Breaker's next move. Hence, property $(b)$ will hold for $k+1$.

If no available edge is adjacent to more than two edges of $B_{k}^{+}(S)$, then $e\left(B_{k}^{+}(S)\right) \leq$ $v\left(B_{k}(S)\right)$. Hence, if there exists an available edge which is incident with two edges of $B_{k}^{+}(S)$, then Maker claims an edge $(x, y)$ that maximizes $\operatorname{deg}_{B_{k}^{+}(S)}(x)+\operatorname{deg}_{B_{k}^{+}(S)}(y)$ among all such edges. It follows that $e\left(B_{k+1}(S)\right) \leq 3$ and $e\left(B_{k+1}^{+}(S)\right) \leq e\left(B_{k}^{+}(S)\right)-$ $2+1 \leq v\left(B_{k}(S)\right)-1=v\left(B_{k+1}(S)\right)+1$ after Breaker's next move. Hence, property (b) will hold for $k+1$.

If every available edge is adjacent to at most one edge of $B_{k}^{+}(S)$, then $e\left(B_{k}^{+}(S)\right) \leq 2$. Maker claims an arbitrary available edge. It follows that $e\left(B_{k+1}(S)\right) \leq 3$ and $e\left(B_{k+1}^{+}(S)\right) \leq e\left(B_{k}^{+}(S)\right)+1 \leq 3 \leq v\left(B_{k}(S)\right)-1=v\left(B_{k+1}(S)\right)+1$ after Breaker's next move. Hence, property $(b)$ will hold for $k+1$.

If property $(b)$ does not hold for $k$, then property $(a)$ must hold. It follows that for every $v \in V\left(G_{k}(S)\right)$, there exists an available edge which is incident with $v$, since the degree of any vertex in $G_{k}(S)$ is $v\left(B_{k}(S)\right)-2$, and there are at most $v\left(B_{k}(S)\right)-3$ edges in $B_{k}(S)$ by assumption. Maker plays as follows (the analysis resembles the one in [2]): 
Case 1.i. (for every $0 \leq i \leq 3$ ). If $e\left(B_{k}^{+}(S)\right) \leq v\left(B_{k}(S)\right)-3-i$, and there is an available edge which is adjacent to at least $3-i$ edges of $B_{k}^{+}(S)$, then Maker claims such an edge, entailing $e\left(B_{k+1}^{+}(S)\right) \leq e\left(B_{k}^{+}(S)\right)-(3-i)+1 \leq v\left(B_{k}(S)\right)-5=v\left(B_{k+1}(S)\right)-3$. It follows that property $(a)$ will hold for $k+1$.

Case 2. If there exists a vertex $v \in V\left(B_{k}(S)\right)$ which is incident with at least 3 edges of $B_{k}^{+}(S)$, then Maker plays according to Case 1.0.

Case 3. Assume that no vertex in $V\left(B_{k}(S)\right)$ is incident with more than 2 edges of $B_{k}^{+}(S)$. By Case 1.3 we may assume that $e\left(B_{k}^{+}(S)\right)>v\left(B_{k}(S)\right)-6$. Maker plays as follows:

Case 3.i. If $e\left(B_{k}^{+}(S)\right)=v\left(B_{k}(S)\right)-5$, then Maker claims an arbitrary available edge which is adjacent to some edge of $B_{k}^{+}(S)$; this is clearly possible as $v\left(B_{k}(S)\right) \geq 6$. It follows by Case 1.2 that property $(a)$ will hold for $k+1$.

Case 3.ii. If $e\left(B_{k}^{+}(S)\right)=v\left(B_{k}(S)\right)-4$ and there is a vertex $v \in V\left(B_{k}(S)\right)$ which is incident with 2 edges of $B_{k}^{+}(S)$, then Maker claims an arbitrary available edge which is incident with $v$. It follows by Case 1.1 that property $(a)$ will hold for $k+1$.

Case 3.iii. If $e^{+}\left(B_{k}(S)\right)=v\left(B_{k}(S)\right)-4$ but no vertex of $B_{k}(S)$ is incident with 2 edges of $B_{k}^{+}(S)$, then the edges of $B_{k}(S)$ must form a matching. Moreover, there are at least two of them as property $(b)$ is not satisfied. In his next move, Maker claims an available edge which is adjacent to two edges of $B_{k}(S)$. It follows by Case 1.1 that property $(a)$ will hold for $k+1$.

Case 3.iv. If $e\left(B_{k}^{+}(S)\right)=v\left(B_{k}(S)\right)-3$, then, by our assumption that property $(b)$ does not hold, it follows that $e\left(B_{k}(S)\right) \geq 4$. Hence, there is at least one vertex $v \in V\left(B_{k}(S)\right)$ that has degree 2 in $B_{k}^{+}(S)$.

If there exists a vertex $v \in V\left(B_{k}(S)\right)$ of degree 2 in $B_{k}^{+}(S)$ which is not incident with any edge of $B_{k}(S)$, then Maker claims an available edge that is incident with $v$ and adjacent to an arbitrary edge of $B_{k}(S)$. It follows by Case 1.0 that property $(a)$ will hold for $k+1$.

If there exists a vertex $w \in V\left(B_{k}(S)\right)$ of degree 2 in $B_{k}^{+}(S)$ which is incident with one edge of $B_{k}^{+}(S) \backslash B_{k}(S)$ and one edge of $B_{k}(S)$, then Maker claims an available edge that is incident with $w$ and is adjacent to some edge of $B_{k}(S)$ which is not incident with $w$. It follows by Case 1.0 that property $(a)$ will hold for $k+1$.

If all vertices of $V\left(B_{k}(S)\right)$ which have degree 2 in $B_{k}^{+}(S)$ are incident only with edges of $B_{k}(S)$, then Maker claims an available edge which is incident with a vertex of degree 2 in $B_{k}^{+}(S)$ and is adjacent to a third edge of $B_{k}^{+}(S)$. We claim that this is always possible. Indeed, it is clear if $e\left(B_{k}^{+}(S) \backslash B_{k}(S)\right)>1$, if $e\left(B_{k}^{+}(S) \backslash B_{k}(S)\right)=1$, then the existence of such an available edge follows since property $(b)$ does not hold and thus $e\left(B_{k}(S)\right) \geq 4$, and if $e\left(B_{k}^{+}(S) \backslash B_{k}(S)\right)=0$, then the existence of such an available edge follows since $4 \leq e\left(B_{k}(S)\right)=$ 
$v\left(B_{k}(S)\right)-3$, and thus $e\left(B_{k}(S)\right) \geq 5$. Either way, it follows by Case 1.0 that property $(a)$ will hold for $k+1$.

This shows, that Maker can ensure that either property $(a)$ or property $(b)$ will hold just before his last move. Since for $k=k_{0}+f-2$ property $(a)$ entails property $(b)$, we conclude that property $(b)$ will hold just before Maker's last move. Hence, there exists some available edge for Maker to claim. Maker plays as follows:

Case 1. If $e\left(B_{k}^{+}(S) \backslash B_{k}(S)\right) \leq 2$, then Maker claims an arbitrary available edge, entailing $e\left(B_{k+1}^{+}(S)\right) \leq e\left(B_{k}^{+}(S) \backslash B_{k}(S)\right)+1 \leq 3$. Hence, we can assume that $e\left(B_{k}^{+}(S) \backslash\right.$ $\left.B_{k}(S)\right) \geq 3$, and thus $e\left(B_{k}(S)\right) \leq 2$.

Case 2. If there exists an available edge which is adjacent to at least three edges of $B_{k}^{+}(S)$, then Maker claims such an edge. It follows that $e\left(B_{k+1}^{+}(S)\right) \leq e\left(B_{k}^{+}(S)\right)-3+1 \leq$ $v\left(B_{k}(S)\right)-1=v\left(B_{k+1}(S)\right)+1=3$. Hence, we can assume that there is no such edge and thus $e\left(B_{k}^{+}(S) \backslash B_{k}(S)\right)=3, e\left(B_{k}(S)\right)=2$, and both edges in $B_{k}(S)$ share an endpoint. Let $a, b$ and $c, d$ denote the endpoints of Maker's paths, and assume without loss of generality that $(b, c)$ and $(b, d)$ are the edges of $B_{k}(S)$.

Case 3. If there exists some edge of $B_{k}^{+}(S) \backslash B_{k}(S)$ which is not incident with $b$, then Maker claims an available edge which is adjacent to such an edge. It follows that $e\left(B_{k+1}^{+}(S)\right) \leq e\left(B_{k}^{+}(S) \backslash B_{k}(S)\right)-1+1 \leq 3$ will hold after Breaker's next move.

Case 4. Otherwise all 5 edges of $B_{k}^{+}(S)$ are incident with $b$. We claim that this cannot happen if Maker follows his strategy. Indeed, let $(e, f)$ denote the last edge claimed by Maker, and assume that Maker's paths in $B_{k-1}(S)$ were $(a, e),(f, b)$ and $(c, d)$ (the other possible case, in which those paths were $(a, b),(c, e)$ and $(f, d)$, can be handled similarly). Since at least 4 of the 5 edges of $B_{k}^{+}(S)$ must have been present in $B_{k-1}^{+}(S)$, we infer that property $(b)$ was satisfied by $B_{k-1}^{+}(S)$. However, by Maker's strategy for this case, it follows that he would not have claimed the edge $(e, f)$, as it does not maximize $\operatorname{deg}_{B_{k}^{+}(S)}(x)+\operatorname{deg}_{B_{k}^{+}(S)}(y)$ in any possible case.

It follows that Maker can indeed build a Hamiltonian path on $K_{n} \backslash S$ in $f-1$ moves, while ensuring that $e\left(B_{k_{0}+f-1}^{+}(S)\right) \leq 3$. This concludes the proof of Lemma 3.5.

\section{Proof of Lemma 3.3}

First, note that if condition (i) from Lemma 3.3 holds, then one can apply Lemma 3.4 to prove that Maker can build a linear forest on $K_{n}[I]$ in $\left\lfloor\frac{|I|}{2}\right\rfloor+1$ moves. This linear forest will consist of $\left\lceil\frac{|I|}{2}\right\rceil-1$ paths, and therefore Maker's graph on $K_{n} \backslash S$ will then consist of $f+\left\lceil\frac{|I|}{2}\right\rceil-1$ paths. It will then follow that

$$
e\left(B_{k_{0}+\left\lfloor\frac{|I|}{2}\right\rfloor+1}^{+}(S)\right) \leq\left(2 f+\left\lceil\frac{|I|}{2}\right\rceil-6\right)+\left(\left\lfloor\frac{|I|}{2}\right\rfloor+1\right) \leq 2\left(f+\left\lceil\frac{|I|}{2}\right\rceil-1\right)-3 .
$$


Hence, without loss of generality we can assume that condition (ii) holds.

Lemma 3.3 now follows directly from Lemma 3.5.

Finally, we need two more short lemmas that will allow us to build the Hamiltonian cycle at the end of Maker's strategy.

Lemma 3.6 Assume that just before Maker's $\left(k_{0}+1\right)$ st move, his graph consists of two spanning vertex disjoint paths $P=p_{1} p_{2} \ldots p_{l}$ and $Q=q_{1} q_{2} \ldots q_{m}$, where $l \geq 4$ and $m \geq 2$. If $e\left(B_{k_{0}}\right)=0$ and there exists a $2<j<l$, such that the edges $\left(p_{1}, p_{j}\right),\left(q_{1}, p_{j-1}\right)$ and $\left(q_{m}, p_{j-1}\right)$ are free, then Maker can build a Hamiltonian cycle in 3 moves.

Proof First Maker claims the edge $\left(p_{1}, p_{j}\right)$. We distinguish between the following two cases:

Breaker claims $\left(p_{l}, q_{b}\right)$ where $b \in\{1, m\}$. In his next move, Maker claims $\left(p_{l}, q_{m+1-b}\right)$. Maker can win by claiming either $\left(p_{1}, q_{b}\right)$ or $\left(p_{j-1}, q_{b}\right)$ in his next move. Clearly, Breaker cannot counter both threats in just one move.

Breaker claims some other edge $e$. Set $b=m$ if $e$ is incident with $q_{m}$, and $b=1$ otherwise. In his next move, Maker claims $\left(p_{l}, q_{b}\right)$. Maker can win by claiming either $\left(p_{1}, q_{m+1-b}\right)$ or $\left(p_{j-1}, q_{m+1-b}\right)$ in his next move. As before, Breaker cannot counter both threats in just one move.

Lemma 3.7 Assume that just before Maker's $\left(k_{0}+1\right)$ st move, his graph consists of a cycle $C$ and a Hamiltonian path $P$ of $K_{n} \backslash C$. If $v(C) \geq 5$ and $e\left(B_{k_{0}}^{+}(C)\right) \leq 3$, then Maker can build a Hamiltonian cycle of $K_{n}$ in 2 moves.

Proof Since $v(C) \geq 5$ and $e\left(B_{k_{0}}^{+}(C)\right) \leq 3$, it follows that there exists a vertex $u \in C$ for which $\left(u, p_{0}\right),\left(v, p_{1}\right),\left(w, p_{1}\right) \notin E\left(B_{k_{0}}^{+}(C)\right)$, where $p_{0}, p_{1}$ are the endpoints of $P$ and $v, w$ are the neighbors of $u$ in $C$. In his $\left(k_{0}+1\right)$ st move, Maker claims the edge $\left(u, p_{0}\right)$. Maker can win by claiming either $\left(v, p_{1}\right)$ or $\left(w, p_{1}\right)$ in his next move. Clearly, Breaker cannot counter both threats in one move. It follows that Maker can indeed win in 2 moves.

We are now ready to prove that Maker can indeed follow his strategy; our proof will follow its phases.

Phase 1. This follows immediately from Lemma 3.1 as $n$ is sufficiently large.

Phases A and B. If at some point during Phase A or Phase B, Maker can close one of his paths into a cycle, then he does so and continues to Phase X. Otherwise, in every move of either phase, Maker tries to connect an endpoint of one of his paths and 
an isolated vertex. It is therefore sufficient to show that there are isolated vertices at any point during those phases. Since Maker cannot close a path into a cycle, the last edge claimed by Breaker must connect the two endpoints of one of Maker's paths. It follows that during Phase A, Maker claims at most one edge for every endpoint of his paths. Since he enters Phase A at most 15 times, and at each such time he has at most 15 paths, it follows that he claims at most $2 \cdot 15^{2}$ edges in Phase A. In Phase B, Maker claims at most one edge for every vertex which is isolated in his graph but not in Breaker's. By Maker's strategy for Phases 1 and 2, there are at most 5 such vertices for every path of Maker and one more since Breaker starts the game. Hence, Maker claims at most $1+5 \cdot 15$ edges in Phase B. Maker also claims $5 \cdot 15$ edges during Phases 1 and 2. It follows that whenever he is in Phase A or in Phase $\mathrm{B}$, there are at most

$$
2 \cdot 15^{2}+1+5 \cdot 15+6 \cdot 15<n
$$

vertices in $K_{n}$ that are not isolated.

Phase 2. Let $S$ denote the set of all vertices of positive degree in Maker's graph. All edges of Breaker are between vertices of $S$ (this was ensured in Phase B). Moreover, $|S|<700$ throughout this phase (recall (1)), and therefore there are more than 12 isolated vertices of $K_{n}$. Hence, Maker can build the required path by Lemma 3.2.

Phase 3. Throughout this phase, unless Maker builds a cycle and goes to Phase X, Breaker claims only edges, both endpoints of which are incident with Maker's paths. It follows that there is always an isolated vertex that Maker can connect to an endpoint of one of his paths.

Phase 4. Let $I$ denote the set of isolated vertices in Maker's graph; it follows from (1), and from Maker's strategy that $|I|=9$ when Maker first enters Phase 4 . Let $S=V\left(K_{n}\right) \backslash I$ and let $k_{0}$ denote the number of moves that Maker has made so far.

First, we have to show that Maker can build the new path $P$ in 8 moves. It follows from Maker's strategy for the previous phases that $e\left(B_{k_{0}}^{+}(S)\right)=0$. Hence, Maker can build a linear forest on $I$ in 5 moves by Lemma 3.4. It is therefore clear that $e\left(B_{k_{0}+5}^{+}(S)\right) \leq 5=v\left(B_{k_{0}+5}(S)\right)-3$, and thus Maker can build a Hamiltonian path on $K_{n}[I]$ within 3 additional moves by Lemma 3.5.

Let $p_{1}, p_{2}$ denote the endpoints of $P$. Since Breaker claims exactly 9 edges during Phase 4, and since there are 15 other paths of Maker, it follows that there is at least one path $Q$ of Maker, with endpoints $q_{1}, q_{2}$, such that both $\left(p_{1}, q_{1}\right)$ and $\left(p_{2}, q_{2}\right)$ are free. Maker claims $\left(p_{1}, q_{1}\right)$ in his last move of this phase.

Phase 5. By Maker's strategy for the previous phases, it is clear that when he first enters Phase 5, his graph consists of exactly 15 paths.

Let $k_{0}$ denote the number of moves that Maker has made so far. Since Breaker has claimed exactly 9 edges during Phase 4, and at least one of these edges connects the 
two endpoints of some path of Maker, it follows by Maker's strategy for the previous phases that $e\left(B_{k_{0}}\right) \leq 8$. Assume that Maker has already made $i \geq 0$ moves in this phase, and has not yet built a cycle. Then, by his strategy, his graph consists of $15-i$ paths, and $e\left(B_{k_{0}+i}\right) \leq \max \{0,8-i\}$. It follows that in his $(i+1)$ st move of this phase, Maker can either close one path into a cycle, or connect two of his paths in the required form.

Phase 6. Just before his last move in Phase 5, Maker had three paths $X=x_{0} x_{1} \ldots x_{a}$, $Y=y_{0} y_{1} \ldots y_{b}$ and $Z=z_{0} z_{1} \ldots z_{c}$. Moreover, all edges connecting endpoints of different paths of Maker were free. Assume without loss of generality that, in his last move of Phase 5, Maker has claimed the edge $\left(x_{a}, y_{0}\right)$. Since we are in Phase 6 (and not in Phase X), it is clear that Breaker has claimed the edge $\left(x_{0}, y_{b}\right)$ in his last move of Phase 5 . It follows that the edges $\left(x_{0}, y_{0}\right),\left(z_{0}, x_{a}\right),\left(z_{c}, x_{a}\right),\left(x_{0}, z_{0}\right)$, $\left(x_{0}, z_{c}\right),\left(y_{b}, z_{0}\right)$ and $\left(y_{b}, z_{c}\right)$ are still free after Breaker's last move. Hence, one can apply Lemma 3.6 to prove that Maker can build a Hamiltonian cycle in exactly 3 moves.

Phase $\mathbf{X}$. Let $k_{X}$ denote the number of moves that Maker has made before entering Phase X. Maker's graph consists at this point of a cycle $C$, and a linear forest $F$ on $K_{n} \backslash C$ that consists of $f \geq 1$ paths. Let $I=V\left(K_{n} \backslash(C \cup F)\right)$. We will show that Maker can build a Hamiltonian path $P$ on $K_{n} \backslash C$ within $n-1-k_{X}$ moves in this phase, such that there are at most 3 edges of Breaker that connect an endpoint of $P$ and a vertex of $C$. It will then follows by Lemma 3.7 that Maker can indeed build a Hamiltonian cycle on $K_{n}$ within $n+1-k_{X}$ moves in this phase.

At any point during Phases 1, A, B or 2, there are at most 12 edges of Breaker, such that at least one endpoint of each of these edges does not belong to a path of Maker. Moreover, if Maker has built the cycle $C$ in one of these Phases, then there may be at most 2 edges of Breaker that are not incident with any vertex of Maker's graph just before Maker's $\left(k_{X}+1\right)$ st move. Indeed, Breaker may have claimed one arbitrary edge just before Maker was able to build his cycle $C$ and one arbitrary edge just after Maker has built his cycle. It follows that

$$
e\left(B_{k_{X}}^{+}(C)\right) \leq 12 \leq\left\lceil\frac{|I|}{2}\right\rceil-6,
$$

because there are clearly more than 50 isolated vertices in Maker's graph during any of these phases.

At any point during Phase 3, if Maker cannot build his cycle, then the endpoints of all of Breaker's edges must be covered by Maker's paths. Moreover, if $(x, y)$ is some edge of Breaker and $x$ is an endpoint of Maker's path $P$, then $y \in V(P)$. If Maker has built his cycle $C$ in Phase 3 , then just before his $\left(k_{X}+1\right)$ st move, there may be at most 2 edges of Breaker that are not incident with any vertex of Maker's graph. Indeed, Breaker may have claimed one arbitrary edge just before Maker was able to 
build his cycle $C$ and one arbitrary edge just after Maker built his cycle. It follows that

$$
e\left(B_{k_{X}}^{+}(C)\right) \leq 2 \leq 2 f+\left\lceil\frac{|I|}{2}\right\rceil-6
$$

because, currently, Maker's graph contains exactly 14 paths. Moreover, it is clear that $|I| \geq 9$ as we are in Phase 3 .

If we are in Phase 5 , then $I=\emptyset$. Let $k_{0}$ denote the number of moves that Maker has made, just before entering Phase 5 for the first time. Recall that $e\left(B_{k_{0}+i}\right) \leq 8-i$ for the first $0 \leq i \leq 8$ moves of this phase (assuming that Maker did not yet build his cycle $C$ ). Thus, if $k_{X}=k_{0}+1+i$ for some $0 \leq i \leq 8$, then

$$
e\left(B_{k_{X}}^{+}(C)\right) \leq 10-i \leq 2(15-i-1)-3
$$

as Breaker may have claimed one arbitrary edge just before Maker has built his cycle, and one arbitrary edge just after Maker has built his cycle. Moreover, Maker has exactly $15-i-1$ paths at this moment.

If Maker has built his cycle $C$ in his $(i+1)$ st move of Phase 5 , for some $9 \leq i \leq 11$, then

$$
e\left(B_{k_{X}}^{+}(C)\right) \leq 2 \leq 2 f-3
$$

as at this moment we have $f \geq 3$.

In all of those cases, we can apply Lemma 3.3 to conclude that Maker can build a Hamiltonian path on $K_{n} \backslash C$, such that there are at most 3 edges of Breaker that connect an endpoint of this path and some vertex of $C$. Moreover, he does so in exactly $n-1-k_{X}$ moves.

Finally, assume that Maker has built his cycle $C$ in Phase 5 such that, just after doing so, his graph on $K_{n} \backslash C$ consists of exactly two paths $P$ and $Q$ (note that Maker has more than two paths when he first enters Phase 5). There are at least two free edges among the four edges that connect the endpoints of $P$ to the endpoints of $Q$, as $e\left(B_{k_{X}}(C)\right) \leq e\left(B_{k_{X}}^{+}(C)\right) \leq 2$. Thus, Maker can claim one of these edges to build a Hamiltonian path on $K_{n} \backslash C$. Moreover, it is clear that $e\left(B_{k_{X}+1}^{+}(C)\right) \leq 3$.

Again, it follows by Lemma 3.3 that Maker can build a Hamiltonian path on $K_{n} \backslash C$, such that there are at most 3 edges of Breaker that connect an endpoint of this path and some vertex of $C$. Moreover, he does so in exactly $n-1-k_{X}$ moves.

This concludes the proof of Theorem 1.1. 


\section{Winning the Hamiltonian cycle game on a small board}

In this section we prove Theorem 1.2. In order to do so, we will present a strategy for Maker and prove that Maker can follow this strategy.

\section{Maker's Strategy:}

Phase 1. Maker builds a path $P=p_{0} p_{1} \ldots p_{4}$ of length 4 , such that just after his 4 th move, all edges of Breaker are incident with some vertex of $P$, and the edge $\left(p_{0}, p_{4}\right)$ is still free. If Maker can close $P$ into a cycle $C$ in his next move, then he does so and continues to Phase 2. Otherwise he adds another edge to the path in his 5th move and builds a cycle $C$ of length at least 5 in his 6 th move. He then proceeds to Phase 2.

Phase 2. Maker builds a Hamiltonian path $Q$ on $K_{n} \backslash C$, while making sure that just before his next move, there are at most three edges of Breaker that connect an endpoint of $Q$ and a vertex of $C$; he then proceeds to Phase 3.

Phase 3. Maker builds a Hamiltonian cycle on $K_{n}$ by connecting the endpoints of $Q$ with two consecutive vertices of $C$.

Proof of correctness In order to prove Theorem 1.2, it suffices to prove that Maker can always follow the aforementioned strategy; our proof will follow its phases.

Phase 1. Since $n>10$, Maker can build the path $P$ with the required properties by Lemma 3.2, with $S=\emptyset$.

If Breaker does not claim $\left(p_{0}, p_{4}\right)$ in his next move, then Maker does and thus builds a cycle $C$ of size 5 .

If on the other hand, Breaker does claim $\left(p_{0}, p_{4}\right)$ in his next move, then Maker claims $\left(p_{4}, p_{5}\right)$, where $p_{5}$ is an arbitrary isolated vertex. Such an isolated vertex exists, as so far, both players have claimed 5 edges each and $n>20$. Since $p_{5}$ was isolated before Maker's 5 th move, it follows that both $\left(p_{0}, p_{5}\right)$ and $\left(p_{1}, p_{5}\right)$ are free after this move. Breaker can claim at most one of these edges in his next move, and therefore Maker can build a cycle $C$ of length either 5 or 6 in his 6 th move.

Phase 2. Let $k \in\{5,6\}$ denote the number of moves that Maker has made during Phase 1. Maker's graph consists at this point of a cycle $C$ and a set $I$ of vertices that are not in $C$. Note that $e\left(B_{k}^{+}(C)\right) \leq 6$. Furthermore, it is straightforward to verify that there are at most 2 edges of Breaker that are not incident with any vertex of $C$. 
As there are at most 6 vertices in the cycle $C$, the set $I$ consists of at least 23 vertices and therefore $\left\lceil\frac{|I|}{2}\right\rceil \geq 12$. This yields

$$
e\left(B_{k}^{+}(C)\right) \leq 6 \leq\left\lceil\frac{|I|}{2}\right\rceil-6
$$

Hence, we can apply Lemma 3.3 to conclude that Maker can build a Hamiltonian path $Q$ on $K_{n} \backslash C$, such that $e\left(B_{k+n-1-v(C)}^{+}(C)\right) \leq 3$.

Phase 3. As $v(C) \geq 5$ and there are at most 3 edges of Breaker that connect an endpoint of $Q$ and a vertex of $C$, we can apply Lemma 3.7 to build a Hamiltonian cycle on $K_{n}$.

This concludes the proof of Theorem 1.2.

\section{Concluding remarks and open problems}

- We have proved that $\kappa\left(\mathcal{H}_{n}^{M}\right) \leq 29$. It would be interesting to determine the value of $\kappa\left(\mathcal{H}_{n}^{M}\right)$ precisely. Using a computer program we have verified that, contrary to Papaioannou's Conjecture, Breaker wins $\mathcal{H}_{n}^{M}$ for every $n \leq 8$. Unfortunately, our program was too slow to analyze $\mathcal{H}_{n}^{M}$ for $n \geq 9$. The interested reader can consult [5].

- A straightforward adaptation of our proof of Theorem 1.2, yields $\kappa\left(\mathcal{H}_{n}\right) \leq 38$ (the only significant difference is that, in Phase 1, instead of using Lemma 3.2 to prove that Maker can build one path with the desired properties, we use Lemma 3.1 to prove that Maker can build two paths). Though this version is different than the one addressed by Papaioannou, it is more useful in practice (see e.g. [3]).

- We have proved that Maker can win the fair Hamiltonian cycle game, played on the edges of $K_{n}$, within $n+1$ moves (and this is best possible). It would be interesting to obtain an analogous result for a biased game (e.g. Breaker claims two edges of $K_{n}$ per move instead of just one), or for a fair game played on some subgraph of $K_{n}$ (e.g. $G(n, 1 / 2))$.

\section{Acknowledgements}

We would like to thank the anonymous referee for his helpful comments. 


\section{References}

[1] V. Chvátal and P. Erdős, Biased positional games, Annals of Discrete Math. 2 (1978) $221-228$.

[2] D. Hefetz, M. Krivelevich, M. Stojaković and T. Szabó, Fast winning strategies in Maker-Breaker games, J. of Combinatorial Theory, Ser. B. 99 (2009), 39-47.

[3] D. Hefetz, M. Krivelevich, M. Stojaković and T. Szabó, Maker-Breaker games on sparse graphs, preprint.

[4] A. Papaioannou, A hamiltonian Game, Annals of Discrete Math. 13 (1982) 171-178.

[5] S. Stich, On two problems regarding the Hamilton cycle game, manuscript.

[6] D. B. West, Introduction to Graph Theory, Prentice Hall, 2001. 